\title{
CLASSIFICATION OF FINITE GROUPS WITH ALL ELEMENTS OF PRIME ORDER
}

\author{
MARIAN DEACONESCU
}

(Communicated by Warren J. Wong)

\begin{abstract}
A finite group having all (nontrivial) elements of prime order must be a $p$-group of exponent $p$, or a nonnilpotent group of order $p^{a} q$, or it is isomorphic to the simple group $A_{5}$.
\end{abstract}

\section{INTRODUCTION}

Let $\mathscr{P}$ be the class of the finite groups having all (nontrivial) elements of prime order. Of course, $\mathscr{P}$ contains the $p$-groups of exponent $p$; but it also contains solvable groups as $A_{4}$ and even the simple group $A_{5}$.

Therefore, a description of the $\mathscr{P}$-groups is not obvious. The aim of this note is to classify these groups. Our notation is standard and conforms to that of [1]. However, we shall denote by $W(G)$ the largest solvable normal subgroup of $G$. All groups are finite. We shall prove the following

Main Theorem. Let $G$ be a $\mathscr{P}$-group. Then one of the following cases occurs:

I . $G$ is a p-group of exponent $p$.

II . (a) $|G|=p^{a} q, 3 \leq p<q, a \geq 3,|F(G)|=p^{a-1},\left|G: G^{\prime}\right|=p$.

(b) $|G|=p^{a} q, 3 \leq q<p, a \geq 1,|F(G)|=\left|G^{\prime}\right|=p^{a}$.

(c) $|G|=2^{a} p, p \geq 3, a \geq 2,|F(G)|=\left|G^{\prime}\right|=2^{a}$.

(d) $|G|=2 p^{a}, p \geq 3, a \geq 1,|F(G)|=\left|G^{\prime}\right|=p^{a}$ and $F(G)$ is elementary abelian.

III . $G \cong A_{5}$.

\section{Preliminary Results}

For the sake of convenience, we list here some of the results used in the proof of the main theorem.

2.1 Let $G$ be a $\mathscr{P}$-group. Then

(i) Every subgroup and every factor group of $G$ is also a $\mathscr{P}$-group.

(ii) If $x \in G$ and $|x|=p$, then $C_{G}(x)$ is a $p$-group of exponent $p$.

Received by the editors March 4, 1988, and, in revised form, November 29, 1988.

1980 Mathematics Subject Classification (1985 Revision). Primary 20E34; Secondary 20D10.

Key words and phrases. p-groups, soluble groups. 
(iii) Every 2-subgroup of $G$ is elementary abelian.

(iv) If $Z(G) \neq 1$, then $G$ is a $p$-group of exponent $p$.

(v) If $G$ is solvable, then there exists $p \in \pi(G)$ such that $F(G)=O_{p}(G)$ and $O_{p^{\prime}}(G)=1$.

(vi) If $G$ is not solvable, then $G / W(G)$ is a simple $\mathscr{P}$-group.

The proofs are trivial.

2.2 ([1, Theorem 5.3.16]). Let $P$ be a $p$-group and let $Q$ be an abelain noncyclic $q$-group of automorphisms of $P$, with $q \neq p$. Then

$$
P=\left\langle C_{P}(x) \mid x \in Q, x \neq 1\right\rangle .
$$

2.3 ([1, Theorem 5.4.10]). If $P$ is a $p$-group without noncyclic abelian subgroups, then $P$ is cyclic or $p=2$ and $p \cong Q_{m}$.

2.4 ([1, Theorem on p. 484]). Let $G$ be a simple nonabelian group with abelian $S_{2}$-subgroups and solvable centralizers of involutions. Then $G \cong \operatorname{PSL}\left(2, p^{n}\right)$, where $p^{n}>3, p^{n} \equiv 3,5 \quad(\bmod 8)$ or $p=2$.

2.5 ([1, Theorem 10.1.4]). Let $\alpha \in \operatorname{Aut}(G)$ such that $|\alpha|=2$ and $C_{G}(\alpha)=1$. Then $G$ is abelian.

2.6 ([2, Satz II 8.3]). There exists a subgroup $U$ of $G=\operatorname{PSL}\left(2, p^{n}\right)$ such that:

(i) $|U|=u=\left(p^{n}-1\right) / k$, where $k=\left(p^{n}-1,2\right)$.

(ii) If $1 \neq x \in U$, then $C_{G}(x)$ is a dihedral group of order $2 u$.

2.7 ([2, Satz II 8.4]). There exists a subgroup $S$ of $G=\operatorname{PSL}\left(2, p^{n}\right)$ such that:

(i) $|S|=s=\left(p^{n}+1\right) / k$, where $k=\left(p^{n}-1,2\right)$.

(ii) If $1 \neq x \in S$, then $C_{G}(x)$ is dihedral of order $2 s$.

2.8 Let $p$ be the least prime divisor of $|G|$ and let $H \neq G$, with $|H|=p$. Then $H \leq Z(G)$. The proof is omitted.

2.9 Let $G$ be a $\mathscr{P}$-group; suppose $G$ solvable and let $p \in \pi(G)$ such that $F(G)=O_{p}(G)$. Let $q \in \pi(G), q \neq p$ and let $Q \in \operatorname{Syl}_{q}(G)$. Then $|Q|=q$.

Proof. Suppose that $T$ is an abelian noncyclic subgroup of $Q$. Since $G$ is solvable, $C_{G}(F(G)) \leq F(G)$, so $C_{F(G)}(x)=1$ for every $1 \neq x \in T$. But then $F(G)=\left\langle C_{F(G)}(x) \mid x \in T, x \neq 1\right\rangle=1$ because of 2.2. This contradiction shows that every abelian subgroup of $Q$ is cyclic. By $2.3, Q$ is cyclic or $Q \cong Q_{m}$ is a generalized quaternion group. The last case gives a contradiction since $Q$ is a $\mathscr{P}$-group by $2.1 \mathrm{i}$ ), while $Q_{m}$ has an element of order 4 . It follows that $Q$ is cyclic, which proves the result because $Q$ is a $\mathscr{P}$-group.

2.10 Let $G$ be a $\mathscr{P}$-group and let $K \neq G$. If $\pi(G) \backslash \pi(K) \neq \varnothing$, then $K \leq F(G)$.

Proof. Let $q \in \pi(G) \backslash \pi(K)$ and let $x \in G$ with $|x|=q$. By 2.1 ii), $x$ acts by conjugation as a fixed-point-free automorphism of $K$ of order $q$. By Thompson's theorem ([1, Theorem 10.2.1]), $K$ is nilpotent. 
2.11 Let $G$ be a solvable, nonnilpotent $\mathscr{P}$-group. Then there exist two distinct primes $p, q$ and a positive integer $a$ such that $|G|=p^{a} q$.

Proof. Let $p \in \pi(G)$ such that $F(G)=O_{p}(G)$. Since $G$ is not a $p$-group, choose $q \in \pi(G), q \neq p$ and let $Q \in \operatorname{Syl}_{q}(G)$, so $|Q|=q$ by 2.9. Set $\bar{G}=G / O_{p}(G)$, so that $O_{p}(\bar{G})=1$ and $O_{p^{\prime}}(\bar{G}) \neq 1$. By $\left.2.1 \mathrm{v}\right)$ applied to the $\mathscr{P}$-group $\bar{G}$, there exists $r \in \pi(G), r \neq p$ such that $O_{p^{\prime}}(\bar{G})=O_{r}(\bar{G})$. Set $K=O_{p, r}(G)$. If $r \neq q$, it follows by 2.10 that $K \leq F(G)=O_{p}(G)$, contradiction. Thus $r=q$ and $|G|=p^{a} q$, as required.

\section{Proof of the Main theorem}

We split the proof into four cases, according to the following possibilities for $G$ : nilpotent, solvable and nonnilpotent, simple nonabelian, nonsolvable and nonsimple. The cases and subcases will follow those in the statement of the main theorem.

I. $G$ is nilpotent. Then by 2.1 iv) $G$ is a $p$-group of exponent $p$ and we are in case I of the statement of the main theorem.

II. $G$ is solvable and nonnilpotent. According to 2.11 , we have $|\pi(G)|=2$ and we have to discuss six subcases.

(a) $2 \notin \pi(G)$ and $p \in \pi(G)$ such that $1 \neq H=F(G)=O_{p}(G) \notin \operatorname{Syl}_{p}(G)$. In this case, let $P \in \operatorname{Syl}_{p}(G)$ and $Q \in \operatorname{Syl}_{q}(G), q \neq p$, so that $|Q|=q$ by 2.9. Set $\bar{G}=G / H$, so $O_{p}(\bar{G})=1, O_{q}(\bar{G})=Q H / H, P / H \in \operatorname{Syl}_{p}(\bar{G})$ and $|P / H|=p$ by 2.9 applied to $\bar{G}$. It follows that $|G|=p^{a} q,|F(G)|=p^{a-1}$ and $\left|G: G^{\prime}\right|=p$. Moreover, $p<q$. Indeed, if $p>q$, then from $\left|O_{q}(\bar{G})\right|=q$ and from 2.8 it follows that $O_{q}(\bar{G}) \leq Z(\bar{G})$. Now by 2.1 (iv) we obtain that $\bar{G}$ is a $q$-group, contradicting $|\bar{G}|=p q$. Thus $p<q$, as asserted.

Finally, we show that $a \geq 3$. One cannot have $a=1$ since $F(G) \neq 1$. On the other hand, if $a=2$, then $|F(G)|=p$ and $F(G)=Z(G)$ by 2.8, which implies by 2.1 (iv) that $G$ is a $p$-group, contradiction. We have verified case II (a) of the main theorem.

(b) $2 \notin \pi(G)$ and $p \in \pi(G)$ such that $F(G)=O_{p}(G) \in \operatorname{Syl}_{p}(G)$. Let $q \in \pi(G), q \neq p$ and let $Q \in \operatorname{Syl}_{q}(G)$, so that $|Q|=q$ by 2.9. If $|G|=p^{a} q$, then $|F(G)|=\left|G^{\prime}\right|=p^{a}$. Suppose that $p<q$ and take $x \in Z(F(G))$ with $|x|=p$. By 2.8 we obtain that $x \in Z(G)$, which implies by 2.1 (iv) that $G$ is a $p$-group, contradiction. Thus $p>q$ and we are in case II (b) of the main theorem.

(c) $2 \in \pi(G)$ and $F(G)=O_{2}(G) \notin \operatorname{Syl}_{2}(G)$. Let $S \in \operatorname{Syl}_{2}(G)$, so $F(G)<S$. By 2.1 (iii), $S$ is elementary abelian, hence $S \leq C_{G}(F(G)) \leq F(G)<S$, contradiction. This case is ruled out.

(d) $2 \in \pi(G)$ and $F(G)=O_{2}(G) \in \operatorname{Syl}_{2}(G)$. Let $p \in \pi(G), p \neq 2$ and let $P \in \operatorname{Syl}_{p}(G)$, so $|P|=p$ by 2.9. Suppose that $|F(G)|=2$. Then by 
2.8, $F(G)=Z(G) \neq 1$ and $G$ is a 2 -group by 2.1 (iv), contradiction. Thus $|F(G)|=2^{a}$, with $a \geq 2$. Since $|G: F(G)|=p$, we are in case II (c) of the main theorem.

(e) $2 \in \pi(G)$ and $1 \neq H=F(G)=O_{p}(G) \notin \operatorname{Syl}_{p}(G), p \neq 2$. Let $S \in$ $\operatorname{Syl}_{2}(G)$ and $P \in \operatorname{Syl}_{p}(G)$, so $H<P$ and $|S|=2$ by 2.9. Set $\bar{G}=G / H$, so that $O_{p}(\bar{G})=1$ and $O_{2}(\bar{G})=S H / H$. But $|S H / H|=2$ and $|P / H|=p$ by 2.9 applied to $\bar{G}$. By $2.8, O_{2}(\bar{G}) \leq Z(\bar{G})$, so $\bar{G}$ is a 2-group by 2.1 (iv). But this contradicts the assumption that $H \notin \operatorname{Syl}_{p}(G)$. This case is ruled out.

$(f) 2 \in \pi(G)$ and $H=F(G)=O_{p}(G) \in \operatorname{Syl}_{p}(G), p \neq 2$. Let $\bar{G}=G / H$, so $O_{p}(\bar{G})=1, O_{2}(\bar{G})=\bar{G}$ by 2.9 applied to $\bar{G}$. Thus $F(G)=G^{\prime}=O_{p}(G)$ and $\left|G: G^{\prime}\right|=2$. Furthermore, let $x \in G$, with $|x|=2$. Since $C_{H}(x)=1$ by 2.1 (ii), $x$ acts by conjugation as a fixed-point-free automorphism of order 2 of $H=F(G)$. By 2.5 it follows that $F(G)$ is an elementary abelian $p$-group and we are in case II (d) of the statement of the main theorem.

III. $G$ is simple nonabelian. Let $x$ be an involution of $G$, so $C_{G}(x)$ is an elementary abelian 2-group by 2.1 (ii) and 2.1 (iii). By $2.4, G \cong \operatorname{PSL}\left(2, p^{n}\right)$, with $p^{n}>3$. If $p=2$, then by $2.7 G$ has an element of order $2^{n}+1$ whose centralizer is a dihedral group of order $2\left(2^{n}+1\right)$, in contradiction with 2.1 (ii).

Thus $p \geq 3$ and by 2.6 and 2.7 there exist elements $a, b \in G$ with $|a|=$ $\left(p^{n}+1\right) / 2,|b|=\left(p^{n}-1\right) / 2$. Since $G$ is a $\mathscr{P}$-group, both $|a|$ and $|b|$ are primes, which forces $|a|=3,|b|=2$ and $p^{n}=5$. Since $\operatorname{PSL}(2,5) \cong A_{5}$, we are in case III of the main theorem.

IV. $G$ is nonsolvable and nonsimple. By $2.1 \mathrm{vi}$ ) and by case III above, one has $G / W(G) \cong A_{5}$, where $W(G) \neq 1$. In any case, since $W(G)$ is a solvable $\mathscr{P}$-group, we know from 2.11 that $|\pi(W(G))| \leq 2$. But $\left|\pi\left(A_{5}\right)\right|=3$, so by 2.10 it follows that $W(G)=F(G)$. Therefore $G / F(G) \cong A_{5}$. Since $F(G) \neq 1$, $F(G)$ is a $p$-group of exponent $p$ by 2.1 (iv). Suppose first that $p=2$. In this case $F(G)$ is an elementary abelian 2-group. Let $S \in \operatorname{Syl}_{2}(G)$, so that $S$ is elementary abelian by 2.1 (iii). Of course, $F(G)<S$ and $|S: F(G)|=4$ since the $S_{2}$-subgroups of $A_{5}$ are isomorphic to the Klein four group $K_{4}$. But $S \leq C_{G}(F(G))$ and since $C_{G}(F(G))=G$ we obtain by the Frattini argument that $G=C_{G}(F(G)) N_{G}(S)$. Since $S$ normalizes both $N_{G}(S)$ and $C_{G}(F(G))$, it follows that $S=G$, contradicting the simplicity of $A_{5}$.

Therefore $p \neq 2$. In this case, if $T \in \operatorname{Syl}_{2}\left(A_{5}\right)$, then $T$ acts as an automorphism group of $F(G)$. Since $T$ is abelian and noncyclic, it follows that $F(G)=\left\langle C_{F(G)}(t) \mid t \in T, t \neq 1\right\rangle$-here we have applied 2.2. But because of 2.1 (ii), we have that $C_{F(G)}(t)=1$ for every $t \in T, t \neq 1$, which shows that $F(G)=1$, a contradiction. This case is ruled out and the proof is now complete.

Remarks. (i) That $A_{5}$ is the only simple nonabelian $\mathscr{P}$-group seems to be a well-known fact. We have included here the proof for completeness. 
(ii) After the first version of this paper was submitted, we learned from [4, p. 290] that G. Zacher has shown in [3] that a solvable nonnilpotent group, having all its nontrivial elements of prime power order, must be of order $p^{a} q^{b}$. Therefore, our lemma 2.11 is a particular (and naturally, more explicit) case of Zacher's theorem.

(iii) In spite of the fact that we have examples of groups satisfying the conditions of case II of the main theorem, we have not been able to see whether these conditions are sufficient for a solvable, nonnilpotent group to be a $\mathscr{P}$-group.

\section{ACKNOWLEDGMENT}

The author is indebted to the referee for a number of improvements on the first version of this note.

\section{REFERENCES}

1. D. Gorenstein, Finite groups, Harper \& Row, 1968.

2. B. Huppert, Endliche Gruppen I, Springer-Verlag, 1967.

3. G. Zacher, Sull'ordine di un gruppo finito risolubile somma dei suoi sottogruppi di Sylow, Rend. Accad. Naz. Lincei, 20 (1956), 171-174.

4. G. Zappa, Fondamenti di teoria dei gruppi II, Edizioni Cremoneze, 1970.

Department of Mathematics, University of Timişoara, 1900 Timişoara, Romania 